\title{
EXPANSIVE HOMEOMORPHISMS WITH THE SHADOWING PROPERTY ON ZERO DIMENSIONAL SPACES
}

\author{
JONG-JIN PARK
}

\begin{abstract}
Let $X=\{a\} \cup\left\{a_{i} \mid i \in \mathbb{N}\right\}$ be a subspace of Euclidean space $E^{2}$ such that $\lim _{i \rightarrow \infty} a_{i}=a$ and $a_{i} \neq a_{j}$ for $i \neq j$. Then it is well known that the space $X$ has no expansive homeomorphisms with the shadowing property. In this paper we show that the set of all expansive homeomorphisms with the shadowing property on the space $Y$ is dense in the space $H(Y)$ of all homeomorphisms on $Y$, where $Y=\{a, b\} \cup\left\{a_{i} \mid i \in \mathbb{Z}\right\}$ is a subspace of $E^{2}$ such that $\lim _{i \rightarrow \infty} a_{i}=b$ and $\lim _{i \rightarrow-\infty} a_{i}=a$ with the following properties; $a_{i} \neq a_{j}$ for $i \neq j$ and $a \neq b$.
\end{abstract}

\section{Introduction}

All spaces considered in this paper are assumed to be compact and metrizable. Let $\Phi$ be a homeomorphism from a space $(X, d)$ onto itself. Given $\delta>0$, a sequence $\left\{x_{i} \mid i \in \mathbb{Z}\right\}$ in $X$ is called a $\delta$-pseudo-orbit of $\Phi$ if

$$
d\left(\Phi\left(x_{i}\right), x_{i+1}\right)<\delta
$$

for every $i \in \mathbb{Z}$. Given $\varepsilon>0$, a sequence $\left\{x_{i} \mid i \in \mathbb{Z}\right\}$ in $X$ is said to be $\varepsilon$-traced by a point $y \in X$ if

$$
d\left(\Phi^{i}(y), x_{i}\right)<\varepsilon
$$

for every $i \in \mathbb{Z}$. We say that $\Phi$ has the shadowing property(or pseudo orbit tracing property) if for every $\varepsilon>0$ there is $\delta>0$ such that every $\delta$-pseudo orbit of $\Phi$ can be $\varepsilon$-traced by a point of $X$. $\Phi$ is called expansive if there is $c>0$ such that for every $x, y \in X$ with $x \neq y$ there is $n \in \mathbb{Z}$ for which

$$
d\left(\Phi^{n}(x), \Phi^{n}(y)\right)>c .
$$

Received March 12, 2004.

2000 Mathematics Subject Classification: Primary 54H20; Secondary 58 F99.

Key words and phrases: expansive homeomorphism, $\delta$ - pseudo-orbit, shadowing property (pseudo orbit tracing property), Dense, Zero dimensional space. 
The constant $c>0$ is called an expansive constant of $\Phi$. For a space $(X, d)$, we denote by $H(X)$ the space of all homeomorphisms of $X$ with the metric

$$
\widetilde{d}(\Phi, \Psi)=\sup \{d(\Phi(x), \Psi(x) \mid x \in X\}
$$

for $\Phi, \Psi \in H(X)$. Let

$$
E(X)=\{\Phi \in H(X) \mid \Phi \text { is expansive }\}
$$

and

$$
P(X)=\{\Phi \in H(X) \mid \Phi \text { has the shadowing property }\} .
$$

Given $\delta>0$ and $a \in X$, we denote a neighborhood $U_{\delta}(a)$ of $a$ by

$$
U_{\delta}(a)=\{b \in X \mid d(a, b)<\delta\} .
$$

Aoki [1] proved that every group automorphism at the Cantor set $C$ has the shadowing property. Sears [5] showed that $E(C)$ is dense in $H(C)$. Dateyma [2] proved that $P(C)$ is dense in $H(C)$ and Kimura [4] also proved the followings.

Proposition. Let $X=\{a\} \cup\left\{a_{i} \mid i \in \mathbb{N}\right\}$ be a subspace of Euclidean space $E^{2}$ such that $\lim _{i \rightarrow \infty} a_{i}=a$ and $a_{i} \neq a_{j}$ for $i \neq j$. Then

a) the set of all expansive homeomorphisms of $X$ is dense in $H(X)$;

b) the set of all homeomorphisms with the shadowing property of $X$ is dense in $H(X)$;

c) $X$ has no expansive homeomorphism with the shadowing property.

A question aries naturally as to whether a zero-dimensional countable compact space admits an expansive homeomorphism with the shadowing property. For the question, Kato and Park [3] shows a zero-dimensional countable compact space admits an expansive homeomorphism with the shadowing property.

In this paper we generalize the result of Kato and Park [3] as in the main theorem of this paper.

\section{The main theorem}

THEOREM 2.1. Let $Y=\{a, b\} \cup\left\{a_{i} \mid i \in \mathbb{Z}\right\}$ be a subspace of Euclidean space $E^{2}$ such that $\lim _{i \rightarrow \infty} a_{i}=b$ and $\lim _{i \rightarrow-\infty} a_{i}=a$, where $a_{i} \neq a_{j}$ for $i \neq j$ and $a \neq b$. Then the set of all expansive homeomorphisms with the shadowing property on $Y$ is dense in $H(Y)$.

Proof. Without loss of generality, we assume that for each $i \in \mathbb{Z}$,

$$
d\left(a_{i+1}, a_{i}\right)<d\left(a_{i}, a_{i-1}\right) \text { if } i \geq 0,
$$


and

$$
d\left(a_{i-1}, a_{i}\right)<d\left(a_{i}, a_{i+1}\right) \text { if } i<0 .
$$

Let $\Psi \in H(Y)$ and $\varepsilon>0$. We construct a homeomorphism $\Phi$ satisfying

$$
\Phi \in E(Y) \cap P(Y) \text { and } \tilde{d}(\Phi, \Psi)<\varepsilon .
$$

To do this, we consider the following two cases:

Case 1. $\Psi(a)=a$ and $\Psi(b)=b$.

Case 2. $\Psi(a)=b$ and $\Psi(b)=a$.

Case 1: For $\varepsilon>0$, we take $n \in \mathbb{N}$ such that for $|i| \geq n, i \in \mathbb{Z}$,

$$
d\left(a_{i}, a\right)<\varepsilon \text { or } d\left(a_{i}, b\right)<\varepsilon .
$$

we define the sets $C_{1}, C_{2}, C_{3}$ by

$$
C_{1}=\left\{a_{i} \mid i \leq-n\right\}, C_{2}=\left\{a_{i} \mid-n<i<n\right\}
$$

and

$$
C_{3}=\left\{a_{i} \mid n \leq i\right\}
$$

Since $\Psi$ is a homeomorphism, there is a $q \in \mathbb{N}$ such that

$$
\Psi\left(a_{i}\right) \in C_{1} \text { for all } i<-q \text { and } \Psi\left(a_{i}\right) \in C_{3} \text { for all } i>q .
$$

Put $k=\max \{q, n\}$. For every $i \in \mathbb{Z}$, we take $l, l^{\prime} \in \mathbb{Z}$ such that

$$
l=\max \left\{i\left|\Psi\left(a_{i}\right)=a_{j},\right| j \mid<k\right\}
$$

and

$$
l^{\prime}=\min \left\{i\left|\Psi\left(a_{i}\right)=a_{j},\right| j \mid<k\right\} .
$$

we define the sets $B, B_{1}, B_{2}$ by

$$
B=\left\{a_{i} \mid l^{\prime} \leq i \leq l\right\} \backslash\left\{a_{i}\left|\Psi\left(a_{i}\right)=a_{j},\right| j \mid<k\right\}
$$

and

$$
B_{1}=\left\{a_{i} \mid \Psi\left(a_{i}\right) \in C_{1}, a_{i} \in B\right\} \text { and } B_{2}=\left\{a_{i} \mid \Psi\left(a_{i}\right) \in C_{3}, a_{i} \in B\right\} .
$$

Then we know that $B=B_{1} \cup B_{2}$.

Define a mapping $\Phi$ from $Y$ onto itself as follows:

i) $\Phi(a)=a$ and $\Phi(b)=b$;

ii) for any $a_{i} \in Y$,

$$
\Phi\left(a_{i}\right)= \begin{cases}\Psi\left(a_{i}\right), & \text { if } i \in\left\{i\left|\Psi\left(a_{i}\right)=a_{j},\right| j \mid<k\right\} \\ a_{i+1}, & \text { if } i \geq l+1 \text { or if } i \leq l^{\prime}-2\end{cases}
$$

iii) $\Phi\left(a_{l^{\prime}-1}\right)$ is a point in the set

$$
\left\{a_{i} \mid i=l^{\prime}, \cdots, l\right\} \backslash\left\{a_{i}|| i \mid<k\right\}
$$


iv) $\Phi\left(a_{i}\right) \in\left\{a_{i} \mid l^{\prime} \leq i \leq-k\right\} \backslash \Phi\left(a_{l^{\prime-1}}\right)$ if $a_{i} \in B_{1}$, $\Phi\left(a_{i}\right) \in\left\{a_{i} \mid k \leq i \leq l+1\right\} \backslash \Phi\left(a_{l^{\prime-1}}\right)$ if $a_{i} \in B_{2}$ and $\Phi\left(a_{i}\right) \neq \Phi\left(a_{j}\right), a_{i} \neq a_{j} \in B_{s}, s=1,2$.

Then $\Phi$ is bijective and $\Phi \in H(Y)$. By the construction of $\Phi$, it is clear that

$$
\tilde{d}(\Phi, \Psi)<\varepsilon .
$$

Now we show that $\Phi$ is expansive. Put

$$
c=\min \left\{d\left(a_{l+1}, a_{l+2}\right), d\left(a_{l^{\prime}-1}, a_{l^{\prime}-2}\right)\right\} .
$$

Then we have

$$
U_{c}\left(a_{l^{\prime}-1}\right)=a_{l^{\prime}-1} \text { and } U_{c}\left(a_{l+1}\right)=a_{l+1} .
$$

Let $x$ and $y$ be two points in $Y \backslash\{a, b\}$ with $x \neq y$. If

$$
x \in\left\{a_{i} \mid l^{\prime}-1 \leq i \leq l+1\right\}
$$

then we get $d(x, y)>c$. Let $x$ be a point in the set $x \in\left\{a_{i} \mid i>\right.$ $l+1\} \cup\left\{a_{i} \mid i<l^{\prime}-1\right\}$. Then we can chose $k, k^{\prime} \in \mathbb{Z}$ such that

$$
f^{k}(x)=a_{l+1} \text { or } f^{k^{\prime}}(x)=a_{l^{\prime}-1} .
$$

Hence $\Phi$ is an expansive homeomorphism. Now we are going to show that $\Phi$ has the shadowing property. Let $\varepsilon_{0}>0$ and let $\varepsilon_{1}=\min \left\{\epsilon_{0}, \varepsilon\right\}$. Take $P, P^{\prime} \in \mathbb{Z}$ with $P^{\prime}<P$ satisfying the following properties:

$$
\text { if } i \leq P^{\prime} \text { then } d\left(a, a_{i}\right)<\varepsilon_{1},
$$

and

$$
\text { if } i \geq P \text { then } d\left(b, a_{i}\right)<\varepsilon_{1} \text {. }
$$

Put

$$
\delta=\min \left\{d\left(a_{p^{\prime}-1}, a_{p^{\prime}-2}\right), d\left(a_{p+1}, a_{p+2}\right)\right\} .
$$

To show that $\Phi$ has the shadowing property, it is sufficient that every $\delta$ pseudo orbit of $\Phi$ can be $\varepsilon_{1}$-traced by a point of $Y$. Let $\zeta=\left\{y_{i} \mid i \in \mathbb{Z}\right\}$ be a $\delta$-pseudo orbit of $\Phi$. we define the sets $A_{1}, A_{2}, A_{3}$ by

$$
A_{1}=\left\{a_{i} \mid i<P^{\prime}\right\}, A_{2}=\left\{a_{i} \mid P^{\prime} \leq i \leq P\right\}
$$

and

$$
A_{3}=\left\{a_{i} \mid i>P\right\}
$$

Then we have the following three possibilities:

1) $\zeta \cap A_{i} \neq \phi$, for each $i=1,2,3$;

2) $\zeta \subset A_{1}$;

3) $\zeta \subset A_{3}$. 
1): If the point $y_{0}$ of the $\delta$-pseudo orbit $\zeta=\left\{y_{i} \mid i \in \mathbb{Z}\right\}$ is in $A_{2}$, then $\zeta$ is $\varepsilon_{1}$-traced by the point $y_{0}$. But if $y_{0} \in A_{1}$ or $y_{0} \in A_{3}$, then we take a point $p$ in the set $\left\{y_{i} \mid i \in \mathbb{Z}\right\} \cap A_{2}$ say $p=y_{r}$. Consider the sequence $\left\{y_{i} \mid i \in \mathbb{Z}\right\}$ and we denote it by $\left\{y_{i-r} \mid i \in \mathbb{Z}\right\}$.

Then the the sequence $\left\{y_{i-r} \mid i \in \mathbb{Z}\right\}$ is $\varepsilon_{1}$-traced by the point $y_{r-r}$.

2): If $\zeta \subset A_{1}$, then it is clear that the $\delta$-pseudo orbit $\zeta=\left\{y_{i} \in\right.$ $\left.A_{1} \mid i \in \mathbb{Z}\right\}$ is $\varepsilon_{1}$-traced by the point $a$.

3): If $\zeta \subset A_{3}$, then it is also obvious that the $\delta$-pseudo orbit $\zeta=$ $\left\{y_{i} \in A_{3} \mid i \in \mathbb{Z}\right\}$ is $\varepsilon_{1}$-traced by the point $b$.

Case 2: Let $\varepsilon>0$ and $l, l^{\prime}$ as in Case 1. Define a mapping $\Phi$ from $Y$ onto itself as follows:

i) $\Phi(a)=b$ and $\Phi(b)=a$;

ii) for any $a_{i} \in Y, \Phi\left(a_{i}\right)=\Psi\left(a_{i}\right)$, if $i \in\left\{i\left|\Psi\left(a_{i}\right)=a_{j},\right| j \mid<k\right\}$;

iii) $\Phi\left(a_{l^{\prime}-1}\right)$ is a point in the set $\left\{a_{i} \mid i=l^{\prime}, \ldots, l\right\} \backslash\left\{a_{i}|| i \mid<k\right\}$;

iv) $\Phi\left(a_{i}\right) \in\left\{a_{i} \mid l^{\prime} \leq i \leq-k\right\} \backslash \phi\left(a_{l^{\prime}-1}\right)$ if $a_{i} \in B_{1}$

$\Phi\left(a_{i}\right) \in\left\{a_{i} \mid k \leq i \leq l+1\right\} \backslash \Phi\left(a_{l^{\prime-1}}\right)$ if $a_{i} \in B_{2}$ and

$\Phi\left(a_{i}\right) \neq \Phi\left(a_{j}\right), a_{i} \neq a_{j} \in B_{s}, s=1,2$

v) $\Phi\left(a_{l+2 t-1}\right)=a_{l^{\prime}-2 t}, t=1,2, \cdots$,

$\Phi\left(a_{l+2 t}\right)=a_{l^{\prime}-2 t+1}, t=1,2, \cdots$,

$\Phi\left(a_{l^{\prime}-2 t}\right)=a_{l+2 t+1}, t=1,2, \cdots$,

$\Phi\left(a_{l^{\prime}-2 t-1}\right)=a_{l+2 t}, t=1,2, \cdots$.

By the same techniques as in the proof of Case 1, we know that $\Phi$ is bijective, $\Phi(a)=b$ and $\Phi(b)=a$. Hence $\Phi \in H(Y)$. By the construction of $\Phi$, we have

$$
\widetilde{d}(\Phi, \Psi)<\varepsilon \text { and } \Phi \in E(Y) \cap P(Y) .
$$

This completes the proof of our main theorem.

\section{References}

[1] N. Aoki, The splitting of zero-dimensional automorphisms and its application, Colloq. Math. 49 (1985), 161-173.

[2] M. Dateyma, Homeomorphisms with the pseudo orbit tracing property of the Cantor set, Tokyo J. Math. 6 (1983), 287-290.

[3] H. Kato and J. Park, Expansive homeomorphisms of countable compacta, Topology Appl. 95(1999), 207-216.

[4] T. Kimura, Homeomorphisms of zero-dimensional spaces, Tsukuba J. Math. 12(1988) no. $2,489-495$.

[5] M. Sears, Expansive self-homeomorphisms of the Cantor set, Math. System Theory. $6(1972), 129-132$. 
Department of Mathematics

Chonbuk National University

Chonbuk 361-763, Korea

E-mail: jjpark46@chonbuk.ac.kr 